the experience of a sighted person. Blindfolding will give some idea of the physical disabilities of the blind but no idea of the heightening of his other senses which he may eventually employ. It cannot reproduce the emotional background. We are so used to "eye to eye" relationships with our fellows that we inevitably feel insecure at our first meeting with someone with whom this is impossible.

As for teaching, I do not foresee any improvement in the situation as less and less time becomes available for specialized teaching of this sort. The task of equipping the family doctor to anticipate the odd ophthalmic disaster is bound to take precedence over training him for all but the most rudimentary aspects of care and rehabilitation of the blind and partiallysighted. Nevertheless, the family practitioner must have confident recourse to others who can supply this expertise, and such others will be grateful to Dr. Shaw for helping their understanding. - I am, etc.,

London N.6

W. J. DiNNING

\section{Vitamin D and Myocardial Infarction}

SIR,-It was most interesting to read in Professor V. Lindén's article (14 September, p. 647) that according to his investigation the consumption of vitamin $\mathrm{D}$ was significantly higher in infarction patients than in control subjects.

As Professor Lindén points out, fish liver is the natural source of vitamin $\mathrm{D}$ in Norway. In Britain the main source is egg yolk, one of the few natural foods in the diet containing vitamin $\mathrm{D}$ in quantity. $\mathrm{My}$ research on the intake of eggs in the United Kingdom ${ }^{1}$ shows a rapid rise in consumption since 1920, apart from short periods of relative deprivation during the 1939-45 war. I have demonstrated that the rise in egg consumption closely follows the rise in mortality from coronary heart disease, and though egg yolk is also a rich source of cholesterol it may be that the presence of vitamin $\mathrm{D}$ in the yolk is an added poten factor associated with coronary heart disease as highlighted by my investigations.

While the aetiology of coronary heart disease is considered to be multifactorial, the potential danger of consuming eggs in quantity should not be overlooked.-I am, etc.,

Community Health Department, Barnsley Area Health Authority,

1 Eskin, F., Community Health, 1971, 2, 179.

\section{Glucagon in Management of Perforated} Oesophagus

SIR,-I read with interest the paper by $\mathrm{Mr}$. O. Daniel and his colleagues (21 September, p. 720) on the use of glucagon in the treatment of acute diverticulitis. I have used it in the management of iatrogenic rupture of the oesophagus in elderly, bad risk patients. Many of these patients are unacoeptable candidates for major surgery and any agent that may assist in the conservative management of the case is of interest.

Glucagon is a potent suppressor of gastric secretion. ${ }^{1}$ Sinoe the initial mediastinitis after perforation of the gullet must be largely due to chemical irritation, any agent that reduces both the quantity and acidity of gastric secretion might be expected to be of benefit. Furthermore, there is evidence that glucagon diminishes gastric motility and also has an antispasmodic effect on the lower oesophageal sphincter. ${ }^{2}$ Reducing motility in the upper gastrointestinal tract should diminish the amount of gastro-oesophageal contents expelled through a perforation.

On this theoretical basis I gave glucagon to three elderly patients with perforated oesophagus. A subclavian central venous line was inserted immediately the diagnosis was made and oral fluids were witheld. Cephalothin was administered in bolus doses of $3 \mathrm{~g}$ every six hours and glucagon was given in an initial dose of $1 \mathrm{mg}$ over five minutes and subsequently at a rate of $2 \mathrm{mg}$ every six hours by continuous infusion. Glucagon was continued until a Gastrografin swallow indicated that the perforation was sealed off. This occurred within four days in one case and within five days in the other two cases. A noteworthy feature of these three cases was that at no time was it necessary to drain the chest. Only minimal amounts of fluid were detected in the pleural cavities.

In the light of these results I suggest that when it has been decided to treat a case of lower oesophageal perforation conservatively glucagon may well contribute to therapeutic success. - I am, etc.,

St. George's Hospital

ROBERT PICKARD

1 Dotevall, G., and Kock, N. G., Gastroenterology, 1963, 45. 364.

2 Jaffer, S. S., et al., Gastroenterology, 1974, 67, 42.

\section{Periodic Fever}

SIR,-At what stage does one stop investigating a patient with recurring bouts of pyrexia and persuade him instead to accept a "diagnosis" of "Periodic Fever"?

A 65-year-old man has suffered from recurring bouts of fever for four years. The temperature rises to $38^{\circ}-39^{\circ} \mathrm{C}$ for 24 hours every five days, with aching in the loins and varying degrees of prostration. All investigations have been negative and there has been no response to antibiotics or to steroids. A chance finding was that the expected spike of fever failed to appear on three occasion following administration of tetanus toxoid. Careful monitoring of temperature has shown accentuation monitoring of temperature has shown accentuation
of fever every 31 days, suggesting that the underof fever every 31 days, suggesting that the under-
lying rhythm is 124 hours, coinciding every 31 lying rhythm is 124 hours, coinciding every 31
days with the normal circadian temperature rise. days with the normal circadian temperature rise.
$\mathrm{A}$ pint $(0.61$.) of blood, removed at the height of the fever, produced a fever when retransfused on the third day, suggesting the presence of a circulating pyrogen.

Reimann and McCloskey, ${ }^{1}$ in a recent report of a case of 23-day fever and mentioning a further instance of 14-day fever, make a plea for the recognition of .periodic disorders due to unknown biorhythms and the avoidance thereby of the unnecessary expense and suffering of prolonged and fruitless investigation.

The purpose of this letter is twofold. Firstly, to seek help: I still feel the need to be aware of the possibility of a microbial cause for fever of five-day rhythm and, if there is one, it may be that some of your erudite readers have knowledge of it. Secondly, to bring to more general notice this extremely trying recurring disorder.

To give the term periodic fever a capital $P$ and a capital $F$ does not of course elevate it to the status of a diagnosis. It may, however, draw attention to a problem still very much in need of elucidation.-I am, etc.,

\section{T. H. BARRIE}

Sherborne, Dorset

1 Reimann, H. A., and McCloskey, R. V., fournal of the American Medical Association, 1974, 228,

\section{Non-union of the Scaphoid}

SIR,--Your leading article (5 October, p. 2) presents a well-balanced and thoughtful appraisal of the problems associated with non-union of the scaphoid. There are, however, two points which merit comment.

While grafting for non-union by the anterior approach has many adherents, it has the disadvantages of not achieving as rigid fixation as is possible by a carefully placed peg graft insertion via the posterolateral approach. This approach is objected to on the grounds of causing possible damage to the blood supply but is there any evidence to indicate that avascular necrosis or other complications have resulted from the use of this approach for the treatment of established non-union? The complication of avascular necrosis of the proximal fragment and semilunar, which is associated very particularly with varying degrees of perilunar transcaphoid dislocation, is not greater following open than closed reduction.

The second comment is that a careful excision of the whole scaphoid has proved a satisfactory procedure in the treatment of late un-united fractures, particularly those associated with osteoarthritic change. It should be emphasized, however, that if through some technical difficulty it is possible to remove only one fragment this should most certainly be the distal fragment, which is the portion affected by secondary arthritic change, the proximal fragment being in no danger in this respect. In all these cperations it is certainly highly desirable to remove the distal $\frac{1}{3}$ in $(8.5 \mathrm{~mm})$ or so of the styloid process of the radius, but wide excision of the styloid process can affect stability.-I am, etc.,

Liverpcol

F. C. DWYER

\section{Management of Lithium Treatment}

SIR,-I feel that in their paper on "Blood Levels and Management of Lithium Treatment" (4 September, p. 650) Dr. J. L. Crammer and his colleagues misrepresent the case as regards the diurnal variations in serum lithium levels. The evidence we have acquired from 23 experiments on patients stabilized for a minimum of 10 weeks on each of the three lithium carbonate preparations on the market is that following each dose as lithium is absorbed from the gastrointestinal tract there is a corresponding rise in the serum lithium level lasting for about two to four hours. Thereafter the level gradually declines over the interdose interval as lithium is eliminated through the kidneys. In the stabilized patient lithium equilibrium is maintained through the daily dose making good the renal lithium loss. The highest serum level found in 200 serum samples was $1.8 \mathrm{mmol} / \mathrm{l}$. and in the vast majority of subjects the level did not fall outside the range $0.5-1.5 \mathrm{mmol} / 1$. at any time during 